\title{
A New Method of Dynamic Prediction on Remaining Useful Life of Barrel
}

\author{
Da XU', a, Ye LUO ${ }^{2, b, ~}{ }^{*}$, Wenbo FAN ${ }^{3, c}$
}

${ }^{1}$ Department of Arms Engineering, Academy of Armored Force Engineering, Beijing, 100072, China ${ }^{2}$ Department of Arms Engineering, Academy of Armored Force Engineering, Beijing, 100072, China

${ }^{3}$ Department of Arms Engineering, Academy of Armored Force Engineering, Beijing, 100072, China

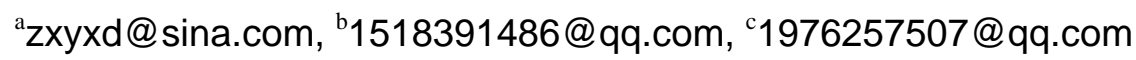

Keywords: remaining useful life; dynamic prediction; artillery barrel

\begin{abstract}
To fully use the performance degradation similarity of the congeneric barrels, for the traits of nonlinear and small sample during the performance degradation process of a barrel, a dynamic prediction model on remaining useful life (RUL) of barrel is proposed, on the basis of fuzzy c-means theory and support vector regression optimized by the optimum model of particle swarm optimization. Lastly, the effectiveness and superiority of this proposed model have been validated by the case of a barrel compared with the improved Verhulst gray theory. In conclusion, a new method is obtained for the prediction on remaining useful life of barrel.
\end{abstract}

\section{Introduction}

During the artillery barrel shooting process, under the combined effect of artillery-bore in high temperature, high pressure, high-speed powder gas and bearing band, the size and structure of the bore are changed, the muzzle velocity and ballistics property of projectile are decreased, thereby affecting the remaining useful life of barrel, which is an important evaluating index of artillery system[1][2].

The performance degradation data of the barrel contains a large number of life related information, based on which can realize the prediction of the remaining useful life of the barrel[3][4]. Currently, there are two ways to analyze the life of the barrel, that is, the modeling method based on mechanism and the modeling method based on data driven. The first method is based on the assumption of ideal state, while the second method requires a lot of data from the same sample to establish the accurate statistical law of life; besides these both can't realize the dynamic prediction of remaining useful life for particular individuals.

This paper proposes a dynamic prediction model of barrel remaining useful life (RUL), on the basis of fuzzy c-means (FCM) theory[5] and support vector regression (SVR) optimized by the optimum model of particle swarm optimization[6][7].Finally, with a barrel as an example, this paper on one hand establishes the degradation path model of specific individual between shot rounds and the muzzle velocity of projectile; on the other hand dynamically updates the degradation path model according to the specific individual real-time measurements, which provided an effective solution to the barrel for dynamic prediction problem of remaining useful life. A new method is provided for the study of remaining useful life on barrel.

\section{Parameter Definition}

$x_{i j}, y_{i j}, \ldots$ represent scalar, where $x_{i j}$ represents the muzzle velocity measurement node of barrel $i$ at the time $j, y_{i j}$ represents the corresponding muzzle velocity measurement results, $\boldsymbol{v}_{k}, \boldsymbol{x}_{i}, \boldsymbol{y}_{i}, \ldots$ represent column vector; $C, D, T, \ldots$ represent special scalar or data sets; $\boldsymbol{U}, \boldsymbol{V}, \boldsymbol{Y}, \ldots$ represent matrix, $\mathbf{Y} \in R^{m \times n}$ is composed by the $y_{i}, i=1,2, \ldots, n$ as columns. For $m_{i}$ represents the total measurement number of barrel $i, D_{i}=\left\{\left(x_{i j}, y_{i j}\right), j=1,2, \ldots, m_{i}\right\}$ represents the degradation data of barrel $\boldsymbol{i}$; 
$D_{z}=\left\{\left(x_{z j}, y_{z j}\right), j=1,2, \ldots, m_{z}\right\}$ represents the historical data of individual barrel; $\bar{x}_{i}, i=1,2, \ldots, m$ represents the standardization measurement node.

\section{Establishment Of Dynamic Prediction Method}

In this paper, the whole process of modeling based on clustering center regression is divided into offline modeling stage and dynamic updating stage.

It is offline modeling stage that according to the performance degradation information of training sample, establishing the degradation path model. This stage is divided into the following 3 steps:

1) To estimate whether the sample data is standard based on the standardization node.

2) If it is standard then clustering analysis based on FCM, or to cluster after normalizing the data. The sample $D_{i}$ is normalized to $\bar{D}_{i}$, the feature vector is marked as $\bar{y}_{i}=\left[\bar{y}_{i 1}, \bar{y}_{i 2}, \ldots, \bar{y}_{i m}\right]$; and the feature vector space is marked as $\boldsymbol{Y}=\left[\overline{\boldsymbol{y}}_{1}, \overline{\boldsymbol{y}}_{2}, \ldots, \overline{\boldsymbol{y}}_{n}\right]$ consisted of $n$ standardization sample. According to the method of FCM[5], the optimal clustering number $\bar{c}$ is carried out by clustering matrix $\boldsymbol{Y}$.

3) To establish the degradation path model of each clustering center. By using $\bar{x}_{i}, \quad i=1,2, \ldots, m$ as input parameter and the clustering center $\hat{\boldsymbol{v}}_{k}(k=1,2, \ldots, \bar{c})$ as output parameter, The degradation path model of each clustering center $\bar{y}_{k}(k=1,2, \ldots, \bar{c})$ is established based on the SVR regression method optimized by PSO[8].

Performance degradation law of specific product is similar with the congeneric products, and the model can be conveniently obtained by weighting the degradation path of congeneric products and dynamically updated and predicted combined with the real-time measurement data. In this paper, the error weighting method is used to measure the similarity of the degeneration path of the congeneric products.

The basic idea of error weighting method[9] is to put the recent measurement node of specific individual into the degradation path model of each clustering center, obtain the degradation estimation value, and determine the weights $\rho_{i}$ according to $e_{k}$ (square of the estimation error), defined in (1), so as to obtain the specific individual degradation path model.

$$
\rho_{i}=\left\{\begin{array}{cc}
0, & e_{i}=0 \text { and } i \neq j \\
\left(\sum_{j=1}^{\bar{c}} \frac{e_{i}}{e_{j}}\right)^{-1}, & e_{i}, e_{j} \neq 0 \\
1, & \text { other }
\end{array}\right.
$$

During learning real-time data, we dynamically update model; on the basis of which to substitute the failure threshold into the model can inversely solve the failure time and achieve dynamic prediction of remaining useful life.

\section{Analysis Of Remaining Useful Life Of A Barrel}

There is rarely dynamic prediction method of barrel remaining useful life at present. Therefore, it can dynamically update the model and barrel RUL predict that applying the model we establish to barrel RUL. Finally we take an artillery barrel as an example by which the effectiveness and superiority of this proposed model have been validated.

The barrel before given to a troop must accept receiving-inspection test, which provides the data basis for life analysis, we take the muzzle velocity attenuation data with the number of projectiles as the performance degradation data and study the barrels in receiving-inspection test (number $1^{\#} \sim 8^{\#}$ ) in this paper. The relationship between muzzle velocity and projectile number are shown in table 1 2, the speed unit is $\mathrm{m} / \mathrm{s}$. 
Table 1 Velocity decline law of barrels $1^{\#} \sim 7^{\#}$

\begin{tabular}{|c|c|c|c|c|c|c|c|}
\hline $\begin{array}{c}\text { Projectile } \\
\text { (round) }\end{array}$ & 0 & 150 & 1530 & 3030 & 5030 & 5530 & 5950 \\
\hline $1^{\#}$ & 976.5 & 977.1 & 968.7 & 960.8 & 943.6 & 941.8 & 937.4 \\
\hline $2^{\#}$ & 971.0 & 975.3 & 961.3 & 949.2 & 931.1 & 926.7 & 917.8 \\
\hline $3^{\#}$ & 975.8 & 978.5 & 968.2 & 960.1 & 954.8 & 944.4 & 927.6 \\
\hline $4^{\#}$ & 968.6 & 970.8 & 967.3 & 955.7 & 942.8 & 934.2 & 924.2 \\
\hline $5^{\#}$ & 962.3 & 972.6 & 963.5 & 954.8 & 942.6 & 938.9 & 930.1 \\
\hline $6^{\#}$ & 976.5 & 978.6 & 958.6 & 952.7 & 943.3 & 937.6 & 923.8 \\
\hline $7^{\#}$ & 973.1 & 976.7 & 954.3 & 957.7 & 942.6 & 935.4 & 928.8 \\
\hline
\end{tabular}

Table 2 Velocity decline law of barrel $8^{\#}$

\begin{tabular}{|c|c|c|c|c|c|c|c|}
\hline $\begin{array}{c}\text { Projectile } \\
\text { (round) }\end{array}$ & 0 & 150 & 1030 & 2530 & 3530 & 4550 & 5730 \\
\hline $8^{\#}$ & 966.7 & 970.1 & 967.3 & 960.4 & 949.2 & 936.6 & 918.2 \\
\hline
\end{tabular}

Barrels $1^{\#} \sim 7^{\#}$ muzzle velocity detection node is [0,150,153, 3030, 503, 5530, 5950]; due to the influence of other test items, the muzzle velocity detection node of barrel $8^{\#}$ is $[0,150,1030,25-30$, $3530,455,5730]$. Barrels $1^{\#} \sim 6^{\#}$ and $8^{\#}$ are selected as the training samples, barrel $7^{\#}$ as the specific individual for RUL prediction and model validation.

To define the measurement node of barrel $1^{\#}$ as the standardization nodes, then only barrel $8^{\#}$ data need to be standardized. The first 5 groups of barrel $8^{\#}$ data are collected as the training set, and the surplus 2 groups as the test set. Firstly, Degradation path models based on PSO-SVR; secondly, range the initialization parameters $(\varepsilon, C, \sigma)$ as $\left[10^{-3} .10\right],[10,100],\left[10^{-2} .10\right]$, optimal parameter value $(\varepsilon, C, \sigma)$ and the normalized data $\bar{x}_{8}$ are got correspondingly as $\left(8.372 \times 10^{-2}, 50,0.447\right)$ and $(966.7$, 970.1, 964.7, 955.2. 92.77, 920.2, 918.8) after the iterative and get. Take $\delta=10^{-5}$, weighted index $q=$ 2 , and substitute Ref. [5] with $\bar{x}_{8} 、 x_{i}(i=1, \ldots, 6)$ to cluster analysis. table 3 means the changing process of clustering validity function values along with the clustering number $c$, the minimum value is obtained at c $=4$, so $\bar{c}=4$, namely the optimal cluster number of the 7 root barrels is 4 , which will make the calculation amount of each step reduced by 3/7.during the dynamic modeling stage.

Table $3 \mathrm{~F}(\boldsymbol{U}$, c) changing law with clustering number c

\begin{tabular}{|c|c|c|c|c|c|}
\hline $\mathrm{C}$ & 2 & 3 & 4 & 5 & 6 \\
\hline $\mathrm{F}(\mathrm{U}, \mathrm{c})$ & 0.0089 & -0.00168 & -0.00238 & -0.0001714 & 0.00689 \\
\hline
\end{tabular}

During the stage, model the respectively degradation path of the four clustering centers obtained in the offline stage based on POS-SVR. Select the prior 4 groups of data of barrel $7^{\#}$ to model, rear 3 groups of data to evaluate the model's accuracy; then gradually increase a group to dynamically update the model, followed by the surplus groups for model checking. After 3 times of dynamic modeling we get the final prediction model, based on which calculate the predictions values of each node. As a comparison, we select the Verhulst gray forecasting method in Ref. [10] to predict the velocity of barrel $7^{\#}$ in the prediction node. The prediction results of the two methods are compared as shown in Table 4. It can be seen that the method used in this paper is more accurate than the Verhulst gray prediction method, the error amplitude is smaller, and the robustness is better.

Table 4 Prediction result comparison of the two means

\begin{tabular}{|c|c|c|c|c|c|c|c|c|}
\hline \multicolumn{2}{|c|}{ Projectile (round) } & 0 & 150 & 1530 & 3030 & 5030 & 5530 & 5950 \\
\hline \multirow{2}{*}{$\begin{array}{c}\text { Verhulst } \\
\text { gray }\end{array}$} & $\begin{array}{c}\text { prediction } \\
\text { s (m/s) }\end{array}$ & 973.1 & 978.2 & 949.4 & 927.6 & 968.6 & 896.4 & 887.7 \\
\cline { 2 - 9 } & error (\%) & 0 & 0.154 & -0.512 & -3.136 & 2.758 & -4.167 & -4.433 \\
\hline \multirow{2}{*}{$\begin{array}{c}\text { This } \\
\text { article }\end{array}$} & $\begin{array}{c}\text { prediction } \\
\text { s (m/s) }\end{array}$ & 965.3 & 974.6 & 956.9 & 954.7 & 946.4 & 936.2 & 924.6 \\
\cline { 2 - 9 } & error (\%) & -0.082 & -0.225 & 0.271 & -0.315 & 0.399 & 0.092 & -0.45 \\
\hline
\end{tabular}




\section{Conclusion}

Since the deficiencies of the traditional barrel RUL prediction method of remaining useful life, we put forward a remaining useful life prediction model of barrel based on FCM clustering center SVR regression method and dynamically update the RUL value of specific barrel. Considering the good generalization ability of FCM to congeneric product information and effective unearthing ability of SVR to small sample, nonlinear characteristics, the congeneric products are clustered by FCM, the life information are standardized and the clustering center degraded path model is established by the SVR method, then the specific individual degradation path model can be got and dynamically updated combined with error weighting method, on the basis of which the failure time can be inversely solved by the failure threshold substituted into the model. The effectiveness of the proposed method is verified by comparing with the Verhulst gray method, which provides a new way to solve the problem of remaining useful life prediction.

\section{Acknowledgement}

In this paper, the research was sponsored by the malitary research project foundation.

\section{References}

[1] Meng X F. Research on life prediction model and bore comprehensive parameters detection system for gun barrel [D]. Nanjing: Nanjing University of Science \& Technology, 2013, 06.

[2] Fan L, Liu E J, Liu W M, et al. Performance decay analysis of a large caliber machine gun barrel in lifetime [J]. Journal of Ballistics, 2013, 32(2): 53-56.

[3] Zhu T W, Wang H L, Chen J. Reliability prediction method of multiple degradations based on unknown degradation paths [J]. Transducer and Microsystem Technologies, 2013, 32(2): 53-56.

[4] Liu J L, Wang H L, Cui X X. Product reliability evaluation on multivariate degradation measures with few data [J]. Electronics Optics \& Control, 2014, 21(2): 77-79.

[5] Zhou S B, Xu W X, Chai T. Data-weighted fuzzy C-means clustering algorithm [J]. Systems Engineering and Electronics, 2014, 30(11): 2314-2319.

[6] Gao R P, Ye S. Improved adaptive pruning algorithm for least squares support vector regression [J]. Systems Engineering and Electronics, 2012, 23(3): 438-444.

[7] Du J G, Wei R H, Liu B P. Nonlinear cointegration test and error correction modeling based on LS-SVM optimized by PSO in small sample[J]. Systems Engineering-Theory \& Practice, 2014, 34(9): 2322-2331.

[8] Wang Y. The research on reliability analysis and prediction of LED street lighting system[D]. Shenyang: Shenyang Ligong University, 2014, 03.

[9] Gebraeel N Z, Lawley M A, Liu R, Parmeshwaran V. residual life predictions from vibration-based degradation signals: a neural network approach [J]. Transactions on Industrial Electonics, 2008, 51(3): 694-700.

[10] Wang F X. Multivariab1e non-equal distance GM(1, m) mode1 and its application[J]. Systems Engineering and Electronics, 2007, 29(3): 388-390. 